\title{
How effective are strategies for non-communicable disease prevention and control in a high risk population in a developing country? Isfahan Healthy Heart Programme
}

Abdolmehdi Baghaei, Nizal Sarrafzadegan, Katayoun Rabiei, Mojgan Gharipour, Ali Akbar Tavasoli, Shahin Shirani, Ahamad Bahonar, Amir Hossein Davarpanah, Mohammad Arash Ramezani, Roya Kelishadi

Isfahan Cardiovascular Research Centre, WHO Collaborating Centre for Research and Training in Cardiovascular Diseases Control, Prevention, and Rehabilitation for Cardiac Patients in the Eastern Mediterranean Region, Isfahan, Iran

Submitted: 28 March 2008

Accepted: 11 August 2008

Arch Med Sci 2010; 6, 1: 24-31

DOI 10.5114/aoms.2010.13503

Copyright (C 2010 Termedia \& Banach

\section{Abstract}

Introduction: The Isfahan Healthy Heart Programme (IHHP) is a communitybased programme for non-communicable diseases prevention and control using both a population and high risk approach in Iran. This study demonstrated the efficacy of IHHP interventional strategies to improve lifestyle behaviours in a population at risk for developing cardiovascular diseases.

Material and methods: Healthy Lifestyle for NCDs High Risk Population is one of ten projects of IHHP. High risk individuals were defined as those who have at least one risk factor for developing coronary artery disease (CAD). Changes of behavioural indicators have been compared between two areas with a survey after 5 years of intervention.

Results: Among high risk individuals in the intervention and reference areas, $77.8 \%$ and $82.5 \%$ had at least one major risk factor for CAD. The prevalence of major risk factors for CAD (except cigarette smoking) was decreased in both intervention and reference areas during 5 years of intervention and the pattern of diet and physical activity was improved.

Conclusions: Interventional activities in IHHP targeting the high risk population seem to be effective in improving lifestyle behaviour, increasing awareness and control of risk factors of the high risk population.

Key words: coronary artery disease, risk factor, healthy lifestyle, community interventions, developing country, Iran.

\section{Introduction}

Cardiovascular disease (CVD) and cerebrovascular disease (CeVD) are the leading causes of mortality and morbidity all around the world [1-3]. In our community, like other developing and developed countries, CVD is the commonest cause of mortality, and has the highest disability-adjusted life year (DALY) rate in the national burden of disease in Iran [4, 5]. Known risk factors are responsible for more than $90 \%$ of coronary events $[6,7]$. Four of them (high blood pressure, diabetes mellitus [DM], obesity and smoking) account for $75 \%$ of leading causes of new cases with coronary artery disease (CAD) [3].
Corresponding author:

Prof. Nizal Sarrafzadegan Isfahan Cardiovascular Research Center Isfahan University of Medical Sciences Address: PO.Box 81465-1148 Isfahan, Iran

Phone: +98 311 3377881-8 Fax: +98 3113373435

E-mail:

nsarrafzadegan@gmail.com 
Most patients with CAD have one or more antecedent known risk factors, so the optimal level of them is associated with very low vascular risk $[3,8]$. Thus it would be necessary that everybody with any risk factors be targeted for appropriate specific strategies for interventions and treatments [9-12]. The major feasible and applicable plan for non-communicable diseases (NCDs) control involves community interventions in an integrated comprehensive approach for CVD risk factor control by using lifestyle modification strategies [13-15].

Some large-scale community integrated programmes for control of NCDs are being conducted by WHO in Europe (CINDI) and America (CARMEN) [16]. These programmes were based on regional programmes, for example the North Karelia Project in Finland. The North Karelia Project was launched in Finland in 1972 in response to a local petition to obtain urgent and effective help to reduce the great burden of exceptionally high coronary heart disease mortality rates in the area. Due to the interventions of this project, the annual mortality rate of CVD was reduced by about 73\% [17].

Isfahan Cardiovascular Research Centre (ICRC), a WHO collaborating centre for CVD prevention, control and management designed a comprehensive integrated community-based interventional programme named the Isfahan Healthy Heart Programme (IHHP), which is a communitybased interventional programme for CAD and control of related risk factors. It represents a strategic shift in public health system programming to: a) enhance the focus on NCDs and their risk factors; b) promote healthy lifestyles using comprehensive population and high risk approaches to prevention [4].

We can control CVD in future, if risk factors are modified now. In this paper, we identify one of the projects that have been conducted for high risk individuals among the study population. The main objective of this project is to analyze the situation of CVD risk factors in a major part of the Iranian population as a developing country and to compare healthy lifestyle improvements between the interventional and reference area.

\section{Material and methods}

The Isfahan Healthy Heart Programme (IHHP), a comprehensive integrated community-based action-oriented study with a reference community, has been conducted by the Isfahan Cardiovascular Research Centre (ICRC, a WHO Collaborating Centre) since 2000 to be completed in 2007 [4].

Two intervention counties (Isfahan and Najafabad) and a reference area (Arak), all located in the central part of Iran, were included in the study. According to the 2000 National Census, the population was 1,895,856 in Isfahan and 275,084 in Najafabad - a county neighbouring Isfahan. Arak, located $375 \mathrm{~km}$ north-west of Isfahan, with a population of 668,531 , was selected as a reference area because of socioeconomic, demographic and health profile similarities to the intervention areas [4].

The programme has 3 phases. The first is a baseline survey for situational analysis, the second is to implement interventional activities in the interventional area, and the last phase will be performed to evaluate the programme output (Outcome Evaluation) [4, 18].

During the second phase we have taken a systematic approach to programme evaluation including each activity (i.e. Process Evaluation) and programme annual monitoring (i.e. Impact Evaluation).

Interventional activities have been designed in 10 different projects to address defined target populations and subjects in an integrated package $[4,18]$. The main objective of the programme is to decrease the incidence of non-communicable diseases (NCDs) including cardiovascular diseases (coronary artery diseases and stroke), diabetes, hypertension (HTN), and cancers, as well as to decrease disability and mortality associated with NCDs [18].

The Healthy Lifestyle for High Risk Population Project is one of 10 that have been designed to apply more specific strategies for lifestyle modification among the high risk population in the community.

\section{Target population}

Adult individuals ( $>19$ years old) with at least one of the following risk factors were included: high blood pressure, diabetes mellitus, dyslipidaemia, metabolic syndrome, obesity and smoking. Also, we included family members of CVD patients as another target group. The main field of intervention was improving knowledge about CVD risk factors and lifestyle modification among target groups (i.e. tobacco control, healthy nutrition, appropriate physical activity).

\section{Baseline survey}

We extracted all people with at least one of the mentioned risk factors, from the main data file of the IHHP baseline survey and study indicators were calculated among them (interventional versus reference communities) (Table I). All measurements and laboratory tests were done using standard protocols [4].

\section{Interventions}

We designed all interventional activities in three parts:

1) Policy/Environmental Strategies,

2) Community Outreach/Programme Services and

3) Surveillance (Table II). 
Table I. Characteristics of IHHP Adult Population Data Files

\begin{tabular}{|lll|}
\hline & Baseline survey & 2005 Survey \\
\hline Sampling method & $\begin{array}{l}\text { Multistage (clustering, random sampling) } \\
\text { population-based }\end{array}$ & $\begin{array}{l}\text { Multistage (clustering, stratified, random } \\
\text { sampling) age and sex based } \boldsymbol{⿰}\end{array}$ \\
\hline Sample size & 6300 in each community & 3000 in each community \\
\hline Indicators & $\bullet$ Demographic characteristics & • Demographic characteristics \\
& - Biochemical indices & BASK about healthy lifestyle, \\
& Physical measurements & NCDs risk factors and CVDs \\
& BASK about healthy lifestyle, NCDs risk factors \\
& and CVDs & \\
& $\bullet$ Awareness, control and treatment indices & \\
\hline
\end{tabular}

IAdopted from CINDI protocol:

- Biochemical indicators: fasting blood sugar, 2-h post-prandial blood sugar, lipid profile (i.e. total cholesterol, HDL cholesterol, LDL cholesterol, triglyceride)

- Physical measurements: blood pressure, weight, height, waist and hip circumference

- BASK: behaviour, attitude, skill and knowledge

- Awareness: individuals who know they have a diseases or risk factor/all individuals who have that

- Treatment index: patients who get medication for their diseases/all patients with that disease

- Control index: patients with controlled disease/all patients with that disease

Table II. Interventional activities for improving cardiovascular risk factors awareness and control and lifestyle modification among high risk populations

\section{Policy/environmental strategies:}

- Encourage work sites to have policies in place that offer regular employee monitoring and education about CVD risk factors control (high blood pressure, dyslipidaemia, diabetes mellitus and obesity) and healthy lifestyle modification (healthy nutrition, physical activity and tobacco control)

- Encourage health insurance companies to offer financial incentives to employees who successfully reduce their modifiable risk factors

- Educate policymakers about the need for supportive environments that provide accessible and low cost cardiovascular medication programmes through hospitals, community health centres, and pharmaceutical companies

- Initiation and development of high risk clinics

\section{Community outreach/Programme services:}

- Promote activities and programmes that offer risk CVD factors screening and education on prevention and control

- Promote opportunities for students to learn about CVD risk factors, and the importance of adopting lifestyle habits to reduce risk

- Increase awareness about CVD risk factors, and the importance of adopting lifestyle habits through work sites, faith-based organizations, and communities

- Encourage all adults to be evaluated based on their individual needs and their health care providers' recommendations

- Encourage all adults to know their family medical history

- Promote lifestyle modification programmes. Examples include weight control, physical activity, healthy eating, and stress management

- Implement campaigns to improve the public's awareness of the linkage between cardiovascular disease risk factors (clustering). Addressed to metabolic syndrome

- Support patient education efforts that increase adherence to medication regimens

- Increase awareness and utilization of programmes offering free or low-cost medications and health care services

- Develop multi-pronged campaigns to promote risk factor reduction messages. Use media channels identified in qualitative research. Examples include grocery bags, buses and other public transportation, local newspapers, radio, and TV

- Work with province health plans and international plans to develop and implement policies and programmes to improve outcomes for all members with CVD risk factors (e.g. world day of high blood pressure)

- Conduct community-based interventions using the outlets identified in qualitative research. Examples include community health centres and other clinics, faith-based organizations, and emergency departments

\section{Surveillance:}

- Monitor the Behavioural Risk Factor Surveillance System estimates for persons ever reporting risk levels 


\section{Strategies}

\section{Population approach strategies}

Interventions have been implemented among the general population via mass media, campaigns, etc. In this approach, interventions have been planned over the general population and target ones received them as members of the community.

\section{High risk (individual) approach strategies}

In this approach, interventions have been implemented among a specific target population in a specific defined environment with more feasibility and availability.

\section{Indirect approach strategies}

In this approach, we have planned some interventions on target populations that have an influence on the main target group (high risk individuals) in the general population. Physicians, nurses and other health care providers are listed in this strategy.

\section{Targets of interventions}

1) All individuals in Isfahan and Najafabad communities with at least one of the mentioned criteria for target groups in this study. There seem to be at least 500,000 persons.

2) Health professionals who have a role in high risk education, management and health care provision.

3) Environments: environments where high risk populations were considered more likely to be found (e.g. some special worksites, hospitals, medical laboratories, retired NGOs).

We followed a comprehensive, integrated approach for our interventions and maintained effective and powerful coordination with all other IHHP projects.

\section{Monitoring}

By extracting a self-reported high risk population from the main data file of the IHHP adult population survey (2005), we studied the BASK indicators (Behaviour, Attitude, Skill and Knowledge) about healthy lifestyle related to NCDs among them to compare with the baseline data. This survey was conducted on an independent sample in two communities to monitor the effects of interventional activities on population lifestyle after 5 years of intervention (Table I).

Data were collected through interview by using a questionnaire including questions about behaviour and knowledge of responders about healthy lifestyle, i.e. dietary and physical activity habits and tobacco use, as well as their relationships to CVD risk factors. A high risk individual has been indicated as one who reported himself or herself about his or her risk factors.

\section{Sampling method and sample size}

We used a multistage sampling design (clustering, stratified, random sampling) to study the indicators in two phases of the IHHP programme (Table I) [4]. Two independent samples were selected in two phases.

All individuals with at least one of the NCDs risk factors among selected adult populations in each community were included in this study to investigate the changes in indicators among high risk populations. About $80 \%$ of the adult population in each community were assigned as high risk individuals and were assessed with respect to their lifestyle indicators (Table III).

Table III. Risk score prevalence of adult population (reference area versus interventional area): IHHP baseline survey

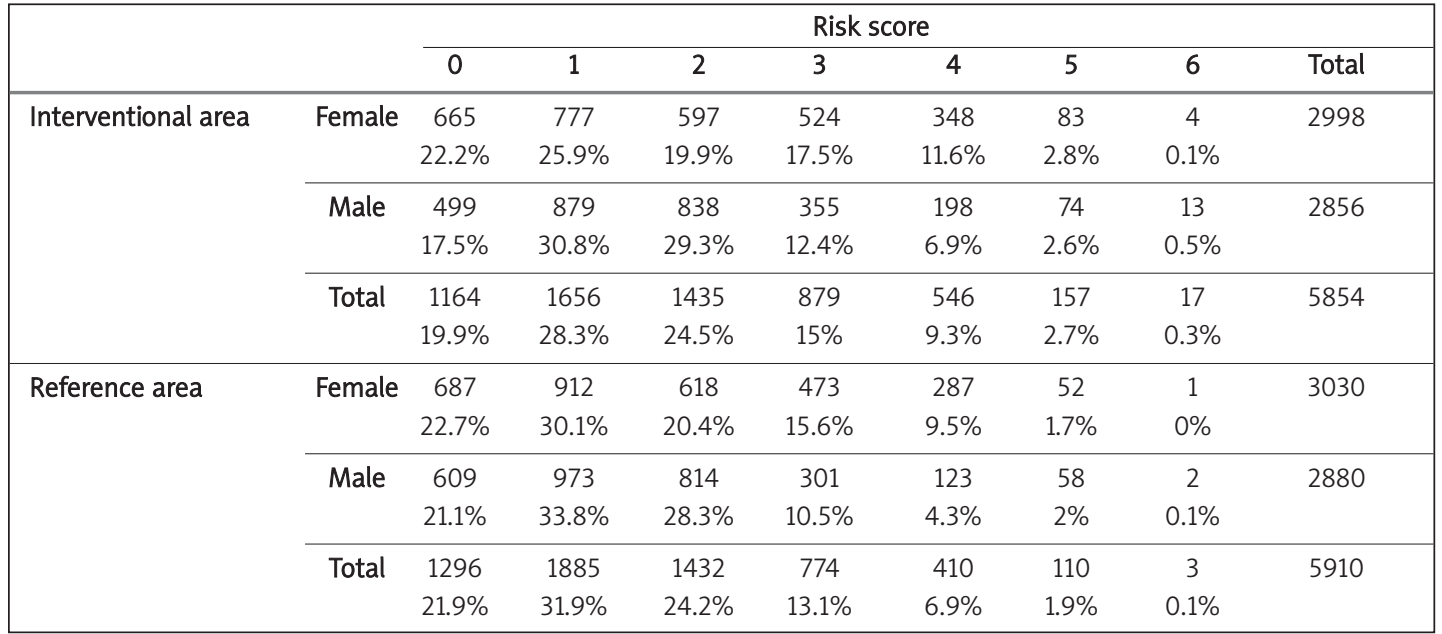

Risk factors included for risk score calculation: high blood pressure, diabetes mellitus, dyslipidaemia, obesity and overweight, smoking, metabolic syndrome 


\section{Statistical analysis}

Statistical analysis was done using SPSS 14 software. Missing analysis was used to process data. All statistical parameters were adjusted based on age according to population pyramids before any analysis. Non-parametric statistical tests were used to test for differences in indicator frequencies between groups (interventional area versus reference area, male versus female) and between two phases. Statistical significant was defined as $p<0.05$.

\section{Results}

\section{Community diagnosis (situational analysis)}

In the baseline survey, about 80 percent of the adult population (9411 from 11764 samples) had at least one CVD risk factor (Table IV). Female to male ratio in the study population was 51:49 in both communities. Mean age of the high risk population in the interventional and reference community was $40.9 \pm 14$ and $41.3 \pm 15$ years, respectively. $79 \%$ of the high risk population in the interventional area and $68 \%$ of the reference area was in an urban area.

In the general view, risk-free individuals were present among females (22.4\%) more than males $(18.8 \%, p<0.05)$ (Table III). The interventional area had fewer risk-free individuals (19.9\%) compared to the reference area $(21.9 \%, p<0.05)$ (Table III).

Overweight or obesity is the most prevalent risk factor (about 50\%) among the total population and diabetes mellitus is the least prevalent (5.6\%) (Table IV). There was observed some discrepancy between risk factor prevalence self-reported by individuals versus documented risk factor prevalence among them (Table IV).

Individuals with diabetes mellitus had the highest score for awareness, treatment and control indices among high risk populations (Table V).

\section{Second survey (2005)}

According to self-reported data from the study population in two communities, $72 \%$ of adults in the interventional area (1779 from 2472) and 65\% of adults in the reference area (1995 from 3070) were included as eligible cases for analysis. We used self-reported data from baseline data to compare the indicators (Table IV).

\section{Behavioural changes}

Healthy lifestyle behaviours have been improved significantly in the high risk population living in the interventional area versus the reference area. Regular daily exercise has been increased by about $45 \%$ among the high risk population in the interventional area (from 15 to $28 \%$ ).

After 5 years of interventions, members of the high risk population in the interventional area have improved their nutritional habits with respect to fruit and vegetable consumption more than the reference area (24\% increasing vs. $5 \%$ increasing, $p<0.05)$. They have used more unsaturated fat and less salt in their diet regimens $(p<0.05)$ (Table VI).

Smoking has been decreased among high risk individuals living in the interventional area more than among those living in the reference area $(p<0.03)($ Table VI).

Table IV. Prevalence of self-reported and documented high risk population in two surveys (2000-2005)

\begin{tabular}{|c|c|c|c|c|c|c|c|}
\hline \multirow[t]{2}{*}{ Risk factor } & \multirow[t]{2}{*}{ Year of survey } & \multicolumn{3}{|c|}{ Interventional area [\%] } & \multicolumn{3}{|c|}{ Reference area [\%] } \\
\hline & & Female & Male & Total & Female & Male & Total \\
\hline \multirow[t]{3}{*}{ Diabetes mellitus } & $2001^{(\mathrm{SR})}$ & 5.1 & 3.9 & 4.5 & 3.5 & 2.4 & 3.1 \\
\hline & $2001^{\mathrm{D}}$ & 7.8 & 6.8 & 7.2 & 7.1 & 5.4 & 6.3 \\
\hline & $2005^{(\mathrm{SR})}$ & 6.3 & 5.9 & 6.1 & 4.2 & 3.1 & 3.6 \\
\hline \multirow[t]{3}{*}{ High blood pressure } & $2001^{(\mathrm{SR})}$ & 11 & 5.4 & 8.3 & 9.3 & 4.9 & 7.1 \\
\hline & $2001^{D}$ & 19.1 & 17.3 & 18.6 & 20.2 & 16.7 & 18.5 \\
\hline & $2005^{(\mathrm{SR})}$ & 15.4 & 10.3 & 13.5 & 9.8 & 7.1 & 8.5 \\
\hline \multirow[t]{3}{*}{ Dyslipidaemia } & $2001^{(\mathrm{SR})}$ & 13.5 & 9.6 & 11.6 & 11.1 & 6.9 & 9.0 \\
\hline & $2001^{D}$ & 23.6 & 21.3 & 21.7 & 20.2 & 19.4 & 19.5 \\
\hline & $2005^{(\mathrm{SR})}$ & 20.9 & 14.8 & 17.9 & 14.6 & 8.8 & 11.7 \\
\hline Obesity and overweight & $2001^{D}$ & 62.6 & 46.4 & 54.7 & 55.8 & 38.4 & 47.3 \\
\hline \multirow[t]{2}{*}{ Smoking } & $2001^{(\mathrm{SR})}$ & 1.3 & 26.1 & 14.9 & 0.8 & 28.1 & 15.2 \\
\hline & $2005^{(S R)}$ & 3.6 & 34.4 & 18.4 & 3.2 & 32.6 & 17.2 \\
\hline Metabolic syndrome & $2001^{D}$ & 37 & 13 & 25 & 33 & 8 & 21 \\
\hline Total number & 2001 & 3169 & 3006 & 6175 & 3222 & 3117 & 6339 \\
\hline of population study & 2005 & 1257 & 1215 & 2472 & 1566 & 1502 & 3070 \\
\hline
\end{tabular}

$S R$ - self-reported, D - documented with biochemical tests or physical examination 
Table V. Awareness, treatment and control indices for diabetes mellitus, dyslipidaemia and high bood pressure among population study (interventional versus reference area): IHHP baseline data

\begin{tabular}{|c|c|c|c|c|c|c|c|}
\hline & \multirow[t]{2}{*}{ Indicator } & \multicolumn{3}{|c|}{ Interventional area [\%] } & \multicolumn{3}{|c|}{ Reference area [\%] } \\
\hline & & Male & Female & Total & Male & Female & Total \\
\hline \multirow[t]{3}{*}{ Diabetes mellitus } & Awareness & 54.3 & 62.3 & 58.5 & 43.4 & 46.1 & 44.7 \\
\hline & Treatment & 31.1 & 38.2 & 34.7 & 27.6 & 29.1 & 28.4 \\
\hline & Control & 19.8 & 22.1 & 20.9 & 14.1 & 17.2 & 15.7 \\
\hline \multirow[t]{3}{*}{ High blood pressure } & Awareness & 30.2 & 55.3 & 43.6 & 28.3 & 43 & 37.1 \\
\hline & Treatment & 21.6 & 41.8 & 32.1 & 17.8 & 33.2 & 25.8 \\
\hline & Control & 8.9 & 24.2 & 16.7 & 7.9 & 15.1 & 11.9 \\
\hline \multirow[t]{3}{*}{ Dyslipidaemia } & Awareness & 38.1 & 48 & 45.4 & 33.5 & 49.8 & 42.1 \\
\hline & Treatment & 16.6 & 28.2 & 23.1 & 12.3 & 28.1 & 19.8 \\
\hline & Control & 9.1 & 15.7 & 12.6 & 7.1 & 13.1 & 10.2 \\
\hline
\end{tabular}

Indicators have significant difference between male and female in all categories $(p<0.01)$

Table VI. Trends of some behavioural changes regarding NCDs risk factors in population with at least one major risk factor $¥$ (interventional vs. reference community: age adjusted)

\begin{tabular}{|c|c|c|c|c|c|c|c|c|}
\hline \multirow[t]{2}{*}{ Subject } & \multirow[t]{2}{*}{ Item } & \multicolumn{3}{|c|}{ Interventional area [\%] } & \multicolumn{3}{|c|}{ Reference area [\%] } & \multirow{2}{*}{$\begin{array}{l}\text { Year of } \\
\text { survey }\end{array}$} \\
\hline & & Male & Female & Total & Male & Female & Total & \\
\hline \multirow[t]{6}{*}{ Nutrition } & Usual unsaturated & 32.5 & 36.8 & 34.3 & 16.8 & 18.1 & 17.6 & 2001 \\
\hline & fat consumerv & 46.2 & 47.3 & 46.9 & 18.3 & 18.5 & 18.4 & 2005 \\
\hline & Added salt to food & 55.7 & 40.4 & 46.1 & 72.1 & 67.3 & 69.3 & 2001 \\
\hline & at the table & 44.8 & 31.9 & 36.9 & 70.4 & 64 & 66.3 & 2005 \\
\hline & Frequent $\circlearrowleft \circlearrowleft$ consumption & 26.7 & 29.4 & 27.8 & 19.7 & 25.9 & 23.7 & 2001 \\
\hline & of fruits and vegetables & 32.3 & 37.4 & 35.2 & 22.5 & 27.4 & 24.7 & 2005 \\
\hline \multirow[t]{6}{*}{ Smoking } & Current smoker & 25.6 & 3.6 & 13.7 & 27.5 & 2.1 & 14.2 & 2001 \\
\hline & & 22.3 & 2.5 & 10.4 & 25.8 & 1.9 & 9.6 & 2005 \\
\hline & Passive smoker & 35.1 & 25.9 & 31.2 & 39.2 & 34.1 & 37.2 & 2001 \\
\hline & & 32.6 & 19.9 & 24.9 & 37.7 & 28.5 & 32.2 & 2005 \\
\hline & Tried to quit & 32.5 & 35.1 & 33.9 & 23.9 & 20.1 & 22.4 & 2001 \\
\hline & in last yeary & 55.7 & 31.3 & 51.6 & 27.9 & 18.2 & 26.8 & 2005 \\
\hline \multirow{2}{*}{$\begin{array}{l}\text { Physical } \\
\text { activity }\end{array}$} & Regular daily exercise & 19.6 & 10.6 & 15.2 & 15.3 & 5.7 & 11.1 & 2001 \\
\hline & & 32.7 & 25.7 & 28.1 & 19.8 & 8.5 & 14.8 & 2005 \\
\hline
\end{tabular}

The data for 2005 were adjusted according to age, because we used an age-based sampling method in this phase. $¥$ Self-reported diabetes mellitus, dyslipidaemia, obesity or high blood pressure

I Use of unsaturated fat is more than saturated fat

ฯ $\circlearrowleft$ Equal or more than 3 times per day

\section{Discussion}

Community interventions in the IHHP programme were successful in improving some lifestyle indicators among high risk groups. The improvement in nutritional habits, smoking and physical activity was significantly higher in the high risk population in the interventional area than in the reference area.

During recent decades, WHO has warranted against NCDs, notably CVD and stroke. It has noted that these diseases have emerged as a public health problem in the developing countries. The IHHP is an ongoing communitybased interventional programme, with one of its objectives being to achieve a decline CVD-related risk factors and intervene to modify lifestyle and reduce risk factors. The current findings are related to the Healthy Lifestyle for High Risk Population Project, which is one of 10 projects of the IHHP. Our results showed that the percentage of adults with no risk factors is very low. Similarly, the percentage of persons maintaining a healthy lifestyle appears to be very low. This is similar to other studies worldwide [19-21]. Mortality due to CVD occurs more in patients with multiple risk factors than risk factors in isolation [22].

In our community, women had a worse CVD risk profile, except for smoking, which was more prevalent among men of both communities (Table IV). 
The prevalence of metabolic syndrome was about 3 times higher in women than in men (Table IV).

Although, similar to other countries in our region, smoking is much more prevalent in men than in women, it is rapidly increasing in women. Although in our community the prevalence of smoking among men is lower than many similar countries in our region [23], this major risk factor for cardiac events must be reduced for NCDs prevention.

Several studies have shown that smokers' risk of developing CAD is 2-4 times that of non-smokers. Cigarette smoking is a powerful independent risk factor for sudden cardiac death in patients with coronary heart disease; even exposure to other people's smoke increases the risk of heart disease, even for non-smokers [24].

Risk factors as a cluster have a multiplicative effect on CVD morbidity and mortality $[25,26]$. In our population, only one fifth of adults did not have CVD risk factors, which is comparable to recent estimates from our region [23].

In our study, during the five years from 2001 to 2005, the behavioural risk factors for CVD have shown a decline in both intervention and reference areas. The pattern of diet and physical activity was improved. The findings of other studies have determined that the intervention programmes reduce CVD risk factors by positively changing nutritional habits, physical activity, and smoking $[15,22]$. However, the major risk factors (HTN, DM, and dyslipidaemia) have increased [15, 22].

Monitoring of self-reported risk factors among populations has been used as an inexpensive and feasible method in other countries [27]. Although the data from self-report monitoring do not have the same validity as documented data, they can be used for trend analysis in surveillance systems [27].

In our study, the higher increase in the prevalence of self-reported risk factors in the interventional area compared to the reference area (Table IV) may be related to an increase in the awareness of individuals living in the interventional area through IHHP educational activities. This can be tested after the final evaluation of IHHP with documented data about CVD risk factors by obtaining blood samples and physical measurements.

Awareness, treatment and control rate of HTN, $\mathrm{DM}$, and hyperlipidaemia determine the distribution of these risk factors. Awareness of HTN in our study was similar to that found in China and Egypt $[28,29]$, but was higher than in Malaysia and Korea $[30,31]$. The treatment rate of HTN in our study was higher than in the aforementioned studies; however, some countries, such as USA, Greece, and France, have reported higher treatment rates than the current study [32-34].

Awareness, treatment and control rates of hyperlipidaemia in our study were lower than other countries, such as China and USA [31, 35, 36]. In our study, more than half of diabetic patients were aware about their disease and most of those aware of having DM were under treatment. There are many methods for monitoring diabetes and glycosylated haemoglobin $\mathrm{A}_{1}\left(\mathrm{HbA}_{1 \mathrm{c}}\right)$ is the most practical one. As we did not check $\mathrm{HbA}_{1 \mathrm{c}}$ in the current study, we cannot assess the control rate in diabetics. However, previous studies in Isfahan have shown that $75 \%$ of patients aged less than 30 years had an ideal to acceptable level of $\mathrm{HbA}_{1 \mathrm{c}}$ and those aged over 30 years had a higher control rate of $82 \%$ [37, 38].

In conclusion, considering the proportions of Iranian adults with multiple cardiac risk factors in this survey, it is important that health decision makers plan interventional activities for controlling modifiable risk factors through lifestyle modifications and pharmacotherapy.

The study highlights the fact that the behavioural basis of CVD, in terms of unhealthy diet, sedentary lifestyle and smoking, is common in our country. Monitoring the burden of disease and factors related to disease risk is a core public health function.

\section{References}

1. Murray CJ, Lopez AD. The global burden of disease: a comprehensive assessment of mortality and disability from diseases, injuries, and risk factors in 1990 and projected to 2020. Boston: Harvard School of Public Health, 1996.

2. Yusuf S, Reddy S, Ounpuu S, Anand S. Global burden of cardiovascular disease. Part I: general considerations, the epidemiologic transition, risk factors and the impact of urbanization. Circulation 2001; 104: 2746-53.

3. Yusuf S, Hawken S, Npuu S, et al. Effect of potentially modifiable risk factors associated with myocardial infarction in 52 countries (the INTERHEART study): casecontrol study. Lancet 2004; 364: 937-52.

4. Sarraf-Zadegan N, Sadri G, Malek Afzali H, et al. Isfahan Healthy Heart Programme: a comprehensive integrated community-based programme for cardiovascular disease prevention and control. Design, methods and initial experience. Acta Cardiol 2003; 58: 309-20.

5. National Burden of Disease study 2003. Burden of diaeses and risk factors in healthy life expectancy in Islamic Republic of Iran. Ministry of health and medical education, Deputy of Health, Nov 2006.

6. Vasan R, Sullivan L, Wilson P, et al. Relative importance of borderline and elevated levels of coronary heart disease risk factors. Ann Intern Med 2005; 142: 393-402.

7. Khot UN, Khot MB, Bajzer CT, et al. Prevalence of conventional risk factors in patients with coronary heart disease. JAMA 2003; 290: 898-904.

8. Stamler J, Stamler R, Neaton JD, et al. Low risk-factor profile and long-term cardiovascular and noncardiovascular mortality and life expectancy: findings for 5 large cohorts of young adult and middle-aged men and women. JAMA 1999; 282: 2012-8.

9. Grundy SM. Primary prevention of coronary heart disease: integrating risk assessment with intervention. Circulation 1999; 100: 988-98.

10. Chobanian AV, Hill M. National Heart, Lung, and Blood Institute Workshop on Sodium and Blood Pressure: 
a critical review of current scientific evidence. Hypertension 2000; 35: 858-63.

11. Canto JG, Iskandrian AE. Major risk factors for cardiovascular disease: debunking the "only 50\%" myth. JAMA 2003; 290: 947-9.

12. Keil U. Coronary artery disease: the role of lipids, hypertension and smoking. Basic Res Cardiol 2000; 95 (Suppl 1): 152-8.

13. Assmann G, Carmena R, Cullen P, et al. Coronary heart disease: reducing the risk: a worldwide view. International Task Force for the Prevention of Coronary Heart Disease. Circulation 1999; 100: 1930-8.

14. Law MR, Wald NJ. Risk factor thresholds: their existence under scrutiny. BMJ 2002; 324: 1570-6.

15. Puska P, Vartianen E, Tuomilehto J, Salomaa V, Nissinn A. Changes in premature deaths in Finland: successful longterm prevention of cardiovascular diseases. Bull WHO 1998; 76: 419-25.

16. World Health Organization. Report on the Consultation on Establishing an Integrated Regional Noncommunicable Disease Network, Cairo, Egypt, 24-26 June 2001. Available in: www.who.int/who-em/NCD/027/E/L.

17. Poska P. Successful prevention of non-communicable diseases: 25 year experiences with North Karelia Project in Finland. Pub Health Med 2002; 4: 5-7.

18. Sarrafzadegan N, Baghaei A, Sadri G, et al. Isfahan Healthy Heart Program: Evaluation of comprehensive, communitybased interventions for non-communicable disease prevention. Prev Control 2006; 2: 73-84.

19. Ford ES, Ford MA, Will JC, Galuska DA, Ballew C. Achieving a healthy life style among United States adults: a long way to go. Ethn Dis 2001; 11: 224-31.

20. Center for Disease Control and Prevention. Prevalence of healthy life style characteristics: Michigan 1998 and 2000. MMWR 2001; 50: 758-61.

21. Greenlund KJ, Zheng ZJ, Keenan NL, et al. Trends in selfreported multiple cardiovascular disease risk factors among adults in the US, 1991-1999. Arch Intern Med 2004; 164: $181-8$

22. Chang M, Mahu RA, Teutsch SM, Hutwagner LC. Multiple risk factors and population attributable risk for ischemic heart disease mortality in the US, 1971-1992. J Clin Epidemiol 2001; 54: 634-44.

23. Maziak W, Rastam S, Mzayek F, Ward KD, Eissenberg T, Keil U. Cardiovascular Health among Adults in Syria: A Model from Developing Countries. Ann Epidemiol 2007; 17: 713-20.

24. Scientific Position. Risk Factors and Coronary Heart Disease. AHA Scientific Statements on Prevention of Coronary Heart Disease and Stroke. 2007.

25. Keil U, Liese AD, Hense HW, et al. Classical risk factors and their impact on incident non-fatal and fatal myocardial infarction and all-cause mortality in southern Germany. Results from the MONICA Augsburg cohort study 1984-1992. Monitoring Trends and Determinants in Cardiovascular Diseases. Eur Heart J 1998; 19: 1197-207.

26. Njolstad I, Arnesen E. Preinfarction blood pressure and smoking are determinants for a fatal outcome of myocardial infarction: a prospective analysis from the Finnmark Study. Arch Intern Med 1998; 158: 1326-32.

27. Greenlund KJ, Zheng ZJ, Keenan NL, et al. Trends in selfreported multiple cardiovascular disease risk factors among adults in the United States, 1991-1999. Arch Intern Med 2004; 164: 181-8.

28. Wang Z, Wu Y, Zhao L, Li Y, Yang J, Zhou B; Cooperative Research Group of the Study on Trends of Cardiovascular Diseases in China and Preventive Strategy for the 21st
Century. Trends in prevalence, awareness, treatment and control of hypertension in the middle-aged population of China, 1992-1998. Hypertens Res 2004; 27: 703-9.

29. Ibrahim MM, Rizk H, Appel LJ, et al. Hypertension prevalence, awareness, treatment, and control in Egypt. Results from the Egyptian National Hypertension Project (NHP). Hypertension 1995; 26: 886-90.

30. Lim TO, Morad Z; Hypertension Study Group. Prevalence, awareness, treatment and control of hypertension in the Malaysian adult population: results from the National Health and Morbidity Survey 1996. Singapore Med J 2004; 45: 20-7.

31. Ford ES, Mokdad AH, Giles WH, Mensah GA. Serum total cholesterol concentrations and awareness, treatment, and control of hypercholesterolemia among US adults: findings from the National Health and Nutrition Examination Survey, 1999 to 2000. Circulation 2003; 107: 2185-9.

32. Stergiou GS, Thomopoulou GC, Skeva II, Mountokalakis TD. Prevalence, awareness, treatment, and control of hypertension in Greece: the Didima study. Am J Hypertens 1999; 12: 959-65.

33. Rywik SL, Davis CE, Pajak A, et al. Poland and U.S. collaborative study on cardiovascular epidemiology hypertension in the community: prevalence, awareness, treatment, and control of hypertension in the Pol-MONICA Project and the U.S. Atherosclerosis Risk in Communities Study. Ann Epidemiol 1998; 8: 3-13.

34. Marques-Vidal P, Evans AE, Cambou JP, et al. Awareness and control of hypertension and hypercholestaerolemia in France and northern Ireland. Q J Med 1997; 90: 341-5.

35. He J, Gu D, Reynolds K, et al. Serum total and lipoprotein cholesterol levels and awareness, treatment, and control of hypercholesterolemia in China. Circulation 2004; 110: 405-11.

36. Frontini MG, Srinivasan SR, Elkasabany A, Berenson GS. Awareness of hypertension and dyslipidemia in a semirural population of young adults: the Bogalusa Heart Study. Prev Med 2003; 36: 398-402.

37. Amini M, Aminoroaya A, Rezvanian H, et al. Glycemic control in diabetic patients in Isfahan. Res Med Sci 1997; 2: 8-12.

38. Abazari P, Amini P, Amini M. Management of diabetic patients in Isfahan. Res Med Sci 2001; 2: 102-5. 